\title{
Computing optimzation methods for 3D real-time computer generated hologram
}

\author{
Tai-zhi Fan \\ College of Information Science \\ Beijing Language University \\ Beijing, 100083, China \\ e-mail: fantz@blcu.edu.cn
}

\begin{abstract}
In recent years, 3D display market becomes more and more exciting. However, the two pictures seen by two eyes will cause the focusing problems of human eyes when watching more than two hours, and furthermore causes the human's uncomfortable. 3D dynamic holographic display can solve all these problems, so it has drawn more and more attentions. However, the huge amount of information cause the computing speed qiute slowly, and it is a challege to speed up the calculation and information processing. In this presentation, we first overview the present computing optimization methods and then present a new method to speed up the computer generated hologram for the use of $3 D$ real-time holographic display.
\end{abstract}

Keywords-computer generation holography, 3D display, realtime hologram, optimization algorithm.

\section{INTRODUCTION}

In recent years, 3D display is more and more popular in the commercial sociaty, especially the $3 \mathrm{D}$ TV is now in the market. The main technology is based on the wearing glasses or the integral image, where each eye sees different 2D pictures and the human brain will combine both pictures into 3D image. It will cause the uncomfortable and other problems. 3D holographic display is regarded to be a perfect display technology by the researchers all over the world[1-5] However, there exist two main open questions. One problem is the resolution of the opto-electronic devices, and the limitation of the space-band-width will cause the smaller size and narrower field of view of 3D image. Many efforts have made to improve the quality of the 3D image. The other is the huge information that composed of the actual 3D scence, and it will cause the heavy computation load for real-time 3D display. Researchers have paid attention to various algorithms for speeding up the generation of the hologram by computer[6-8]. In this paper, we descibe the state-in-art algorithms and present a simple method for generating the computer generated hologram(CGH).

\section{BASIC PRINCIPLE}

There are two main methods for light propagation and CGH generation. One is point source method and the other is the polygon plane method.

\section{A. Point Source Method}

The postion of the pth object point can be $\left(x_{p}, y_{p}, z_{p}\right)$,and the amplitude and the phase are descibed as $O_{p}$ and $\varphi_{p}$. If the objective is random scattering, the phase could be random $\varphi_{p}$.

Set hologram in the plane of $z=0$, the complex amplitude distribution $O(x, y)$ of the objective light wave can be written as

$$
O(x, y)=\sum_{p=1}^{N} \frac{O_{p}}{r_{p}} \exp \left[j\left(k r_{p}+\phi_{p}\right)\right],
$$

where $\mathrm{N}$ denotes the number of the sampling points, the distance between the pth objective point and the hologram sampling point $(x, y, z)$ is described as

$$
r_{p}=\sqrt{\left(x-x_{p}\right)^{2}+\left(y-y_{p}\right)^{2}+z_{p}^{2}} .
$$

The complex amplitude $R(x, y)$ of the reference light wave can be written as

$$
R(x, y)=R_{0} \exp \left[j\left(k x \sin \theta_{R}\right)\right],
$$

where $R_{0}$ and $\theta_{R}$ stand for the amplitude and the angle of the reference light wave. Therefore the complex amplitude distribution of the hologram can be written as

$$
H(x, y)=\sum_{p=1}^{N} \frac{O_{p}}{r_{p}} \exp \left[j\left(k r_{p}+k x \sin \theta_{R}+\phi_{p}\right)\right]
$$

The algorithms for the point source method are based on Eq.(4), and sometimes Taiyor expasion is used for further apprioximation and simplification, sometimes the look up table is employed to speed up the caculations. Principle of the point source method can be shown in Fig. 1(a), and corresponding holograms for each point can be described in Fig. 1(b).
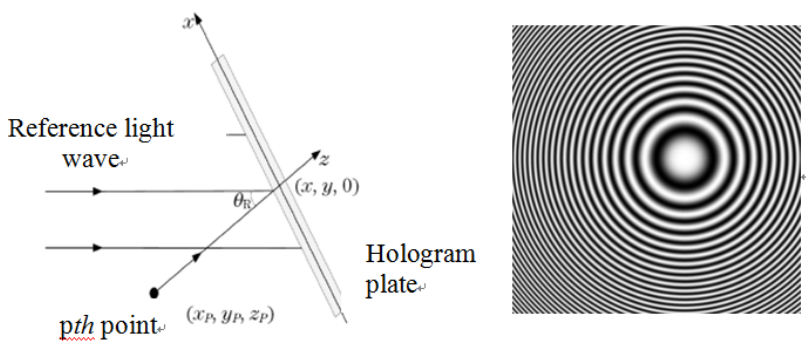

Fig. 1 The schematic view of the point source method for hologram generation.(a)Principle and (b)hologram. 


\section{B. Polygon Plane Method}

Polygon plane method is based on the plane-wave spectrum theory and the sperical-wave diffraction theory. The acutual 3D object can be divided into many planes, and each plane of the 3D object is not parallel to the plane of the hologram. The plane-wave spectrum theory can deal with the propagation from one plane to the other plane. It should deal with the micro-polygon plane, and the propagation direction should be in accordance with the normal direction of the hologram. Therefore the real polygon plane should be rotated, shifted, and transferred before the calculation. It can be shown in Fig. 2(a), The transfermation of between the global coordinates $(x, y, z)$ and local coordinates $(\hat{x}, \hat{y}, \hat{z})$ is $k=T^{-1} \hat{k}$, and the matrix can be written as

$$
\mathbf{T}^{-1}=\left[\begin{array}{lll}
a_{1} & a_{2} & a_{3} \\
a_{4} & a_{5} & a_{6} \\
a_{7} & a_{8} & a_{9}
\end{array}\right]
$$

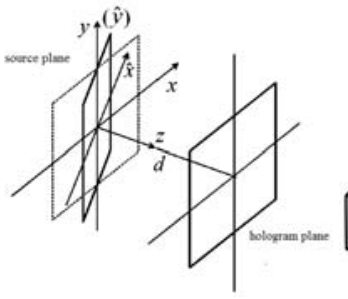

(a )

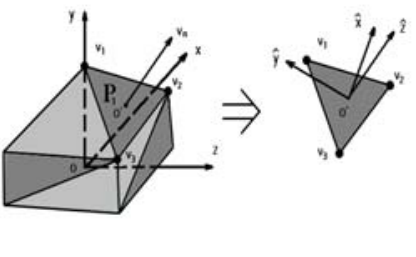

(b)
Fig. 2 Schematic view of (a) polygo-plane spectrum theory and (b) global coordinate and local coordinate.

The complex amplitude can be expressed as the spectrum of the micro-plane in the global coordinates

$$
\begin{aligned}
E(x, y) & =\mathscr{F}^{-1}\{e(\hat{u}, \hat{v})\} \\
& =\iint_{-\infty}^{\infty} e(\hat{u}, \hat{v}) \exp [i 2 \pi(\hat{u} \hat{x}+\hat{v} \hat{y})] d \hat{u} d \hat{v} \\
& =\iint_{-\infty}^{\infty} e[\hat{u}(u, v), \hat{v}(u, v)] \exp \{i 2 \pi[\hat{u}(u, v) x+\hat{v}(u, v) y]\} J(u, v) d u d v
\end{aligned}
$$

where $\hat{u}$ and $\hat{v}$ denote the space frequceies in the local coordinates $\hat{x}$ and $\hat{y}$, respectively, and $J(u, v)$ is Jaccobi matrix that can be defined as

$$
\begin{aligned}
J(u, v) & =\frac{\hat{u}(u, v)}{u} \frac{\hat{v}(u, v)}{v}-\frac{\hat{u}(u, v)}{v} \frac{\hat{v}(u, v)}{u} \\
& =\left(a_{2} a_{6}-a_{3} a_{5}\right) \frac{u}{w(u, v)}+\left(a_{3} a_{4}-a_{1} a_{6}\right) \frac{v}{w(u, v)}+\left(a_{1} a_{5}-a_{2} a_{4}\right)
\end{aligned}
$$

According to the spectrum propagation theory, the complex amplitude distribution in the hologram can be discribed as

$$
\begin{aligned}
H(x, y)= & \iint_{-\infty}^{\infty} h(u, v) \exp [j 2 \pi(u x+v y)] d u d v \\
= & \iint_{-\infty}^{\infty} e[\hat{u}(u, v), \hat{v}(u, v)] \exp \{i 2 \pi[\hat{u}(u, v) x+\hat{v}(u, v) y]\} \\
& J(u, v) \exp \left(j k d \sqrt{1-\lambda^{2} u^{2}-\lambda^{2} v^{2}}\right) d u d v
\end{aligned}
$$

Because this method will introduce deviation error because of the discrete Fourier Transformation,and the calculation time is quite long. Furthermore, the discrete data will cause calculation error as well. Further improvement method is presented by analytical method, and it can save time. In this presentation, we present a method to speed up the calculation.

\section{NUMERICAL AND EXPERIMENTAL RESULTS}

The simulated results are in Fig. 3(a)-(f), where (a) illustrates 3D cubic module, and it takes $91.2188 \mathrm{~ms}$ to calculate; (b) stands for 3D cubic with surface labeled characters, and it takes $93.8594 \mathrm{~ms}$ to compute; (c) denotes 3D cubic with surface symbol, and it costs $92.4189 \mathrm{~ms}$; (d) represents 3D triangle module, and it takes 53.8125ms; (e) illuminates the focused plane in the front and (f) the focused plane in the back.

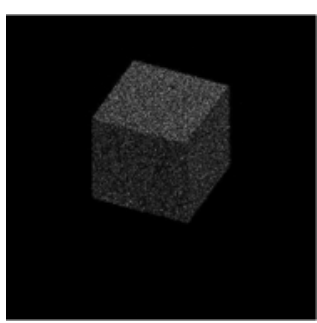

(a)

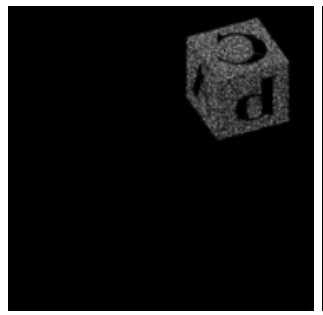

(c)

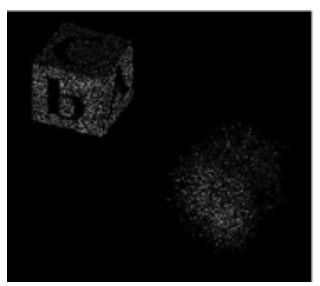

(e)

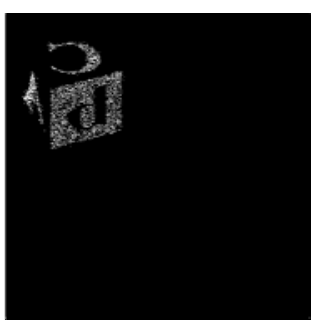

(b)

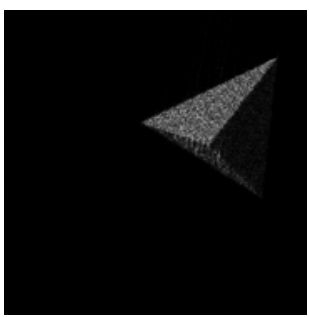

(d)

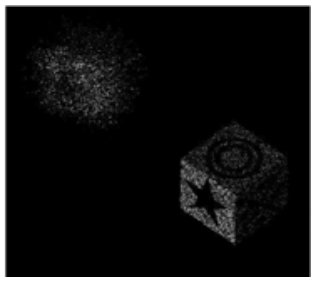

(f)
Fig. 3 Simulated numerical results.(a)3D cubic module, (b) 3D cubic with surface labeled characters,(c) 3D cubic with surface symbol,(d)3D triangle module,(e)focused in the front, and (f) focused in the back. 
Table 1 Compter time used for the program

\begin{tabular}{|c|c|c|c|}
\hline $\begin{array}{c}\text { Computed } \\
\text { part }\end{array}$ & $\begin{array}{c}\text { Paramenters } \\
\text { set }\end{array}$ & $\begin{array}{c}\text { Micro-plane } \\
\text { position } \\
\text { reading }\end{array}$ & $\begin{array}{c}\text { Transferatio } \\
\text { n to local } \\
\text { coordinates }\end{array}$ \\
\hline $\begin{array}{c}\text { Computer } \\
\text { time (ms) }\end{array}$ & 0.0469 & 0.0313 & 0.0469 \\
\hline $\begin{array}{c}\text { Computer } \\
\text { part }\end{array}$ & $\begin{array}{c}\text { Compute } \\
\text { the local } \\
\text { spectrum }\end{array}$ & $\begin{array}{c}\text { Spectrum } \\
\text { rotation }\end{array}$ & CGH \\
generation
\end{tabular}

Table 1 is the computer time for the calculation of the CGH. In the spectrum rotation, the interpolation time is $3.1250 \mathrm{~ms}$ 。 It can be seen that the generation of the microplane occupies $73 \%$ of the total time, and the interpolation time occupies $20 \%$ total time. Therefore, if both calculation speed can be accelerated, the total time could be speed up.

The experiment is performed, and the optical experimental results are shown in Fig. 4(a) and (b).

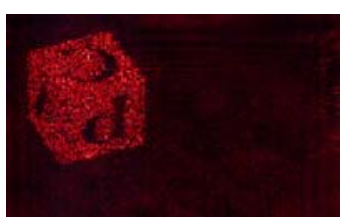

(a)

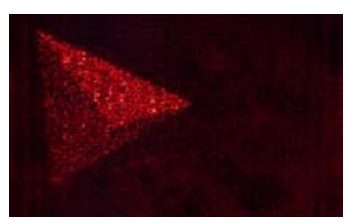

(b)
Fig. 4 The optical experimental results.

\section{CONCLUSION}

In brief, we first review the present computing optimization methods and then present a new method to speed up the computer generated hologram for the use of 3D real-time holographic display. The numerical results and the optical experimental results are in good agreement and it is a good method to speed up the generation of CGH efficiently.

\section{ACKNOWLEDGMENT}

This work is supported by the National Natural Science Founding of China (61077007).

\section{REFERENCES}

[1] D. F. McAllister, Stereo Computer Graphics and Other True 3-D Technologies, Princeton University Press, 1995, pp.30-55.

[2] S. Pastoor, J. Liu, S. Renault, “An experimental multimedia system allowing 3-D visualization andeye-controlled interaction without user-worn devices”, IEEE Trans. on Mulitmedia, vol. 1(1), pp.41-52, 1999.

[3] S. A. Benton, V. Michael Bove Jr., Holographic Imaging, New York Wiley-Interscience, 2008, pp.22-32.

[4] C. Slinger, C. Cameron, M. Stanley, "Computer-generated holography as a generic display technology”, Computer, vol.38, pp.46-53, 2005.

[5] Y. Pan, X. Xu, S. Solanki, "Fast CGH computation using S-LUT on GPU”, Optics Express, vol.17(21), pp.18543-18555, 2011.

[6] S.-C. Kim, E.-S. Kim, "Computational approaches for fast generation of digital 3D video holograms”, CHINESE OPTICS LETTERS, vol.7(12), pp.1083-1091, 2009.

[7] Y. Liu, J. Dong, Y. Pu, "High-speed full analytical holographic computations for true-life scenes”, OPTICS EXPRESS, vol.18(4), pp.3345-3351, 2010.

[8] L. B. Lesem, P. M. Hirsch, J. A. Jordan, "The Kinoform: A New Wavefront Reconstruction Device", Journal of Research and Development (IBM), vol. 13 (2), pp.150-152,1969. 